\title{
Direct Imaging of Incoherent-to-Coherent Structural Dynamics in Plasmonic Nanorods with Ultrafast Electron Microscopy
}

\author{
Ryan A. Gnabasik ${ }^{1}$, David T. Valley ${ }^{1}$, Matthew K. Quan ${ }^{1}$, Vivian E. Ferry ${ }^{1}$ and David J. Flannigan ${ }^{1 *}$ \\ 1. Department of Chemical Engineering and Materials Science, University of Minnesota, Minneapolis, \\ MN 55455, USA \\ * Corresponding author: flan0076@umn.edu
}

Ultrafast photoexcitation of plasmonic metal nanostructures results in a cascade of incoherent and coherent electronic, optical, and structural responses along discrete temporal trajectories. Surface plasmon excitation and rapid electron thermalization couple to coherent, low-frequency acoustic modes over the span of picoseconds, and the precise nature of the coupling and mode excitation are sensitively dependent upon particle-particle and particle-substrate interactions, geometric shape, and elastic properties [1-4]. Importantly, the electronic and structural responses are interdependent, as the atomic and nanoscale transient elastic distortions modulate the dielectric response function, which in turn modifies the surface plasmon resonance, while dependence of plasmonic responses on the proximity and the configuration of neighboring particles could potentially affect the amplitudes of the coherent modes. In light of this complexity, detailed insights into the temporally- and spatially-dependent responses in imperfectly-arranged plasmonic assemblies may be gained via direct-imaging methods capable of accessing combined nanometer-picosecond scales for single-particle measurements [4]. Among a variety of time-resolved spectroscopic and structural probes, ultrafast electron microscopy (UEM) has been used to directly image discrete, nanoscale structural dynamics in plasmonic single-particles and few-particle clusters [5]. With UEM, the onset of specific acoustic modes was imaged, and the excitation of isolated, nanoscale vibrational hot spots at discrete particle-particle contact points was observed using Fourier frequency maps generated from the real-space picosecond image series. These vibrational hot spots match the spatial positions of the plasmonic hot spots generated from concentrated electromagnetic fields.

Here, we report the direct imaging with UEM of photoexcited incoherent-to-coherent structural dynamics in a randomly-oriented cluster of plasmonic Au nanorods supported by an amorphous silicon nitride substrate. With picosecond bright-field imaging, the atomic-to-nanoscale structural response of the individual particles comprising the cluster is spatially resolved and tracked over the first 100 ps following fs in situ photoexcitation. An initial increase in contrast strength (i.e., decrease in counts) within the particles is observed immediately during and after photoexcitation. The instrument-limited duration of the couple-percent reduction in counts occurs at approximately the same time for all particles in the cluster, indicative of an ultrafast rise in thermal diffuse scattering due to electron-lattice thermalization and an increase in amplitude of incoherent atomic mean-square displacements. Following this, emergence of coherent, low-frequency acoustic modes is observed, with clear structural oscillations observable in the particles. Interestingly, using a space-time contour-plot analysis, we find there to be a delay of 12 ps between the commencement of the observable electron-lattice thermalization and the onset of the first acoustic-phonon period within a particularly responsive particle. Upon launch, the acoustic mode propagates across the particle at roughly the speed of sound and encounters a counterpropagating disturbance near the center of the structure. In addition to the experimental configuration and the results, proposals for the mechanisms underlying the observed behaviors will be presented [6]. 
[1] M Pelton et al., Phys. Rev. B 73 (2006), p. 155419.

[2] H Baida et al., Phys. Rev. Lett. 107 (2011), p. 057402.

[3] WS Chang et al., Nat. Commun. 6 (2015), p. 7022.

[4] G Beane et al., Rep. Prog. Phys. 82 (2019), p. 016401.

[5] DT Valley, VE Ferry and DJ Flannigan, Nano Lett. 16 (2016), p. 7302.

[6] This work was supported primarily by the National Science Foundation through the University of Minnesota MRSEC under Award Number DMR-1420013.
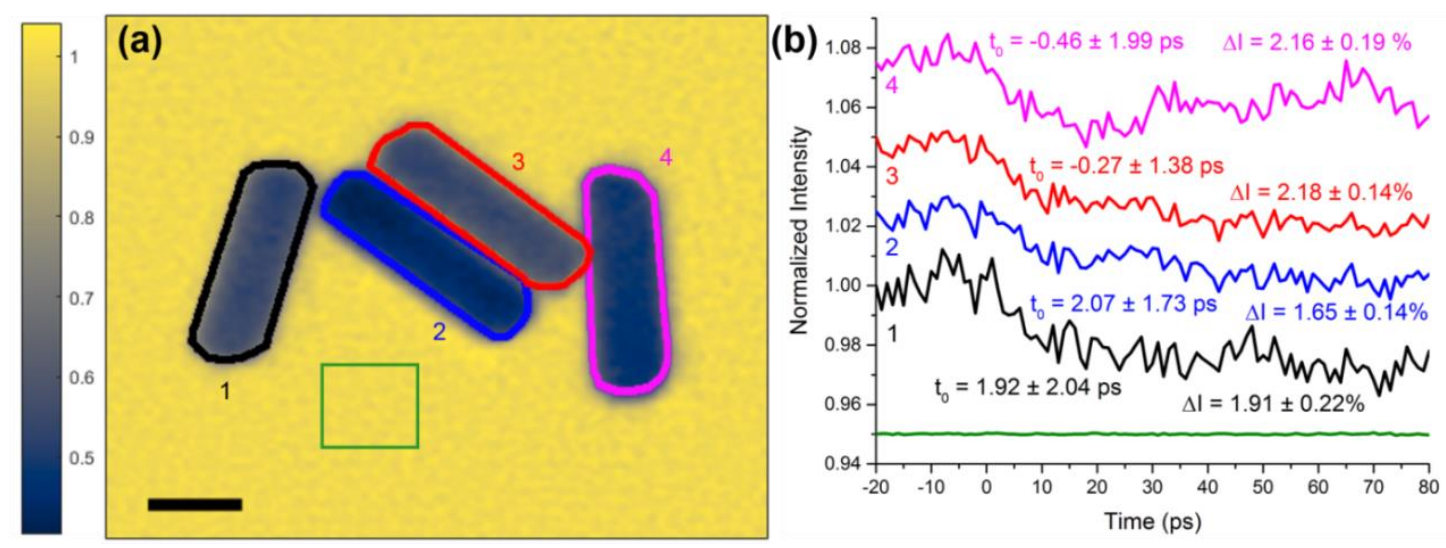

(c)

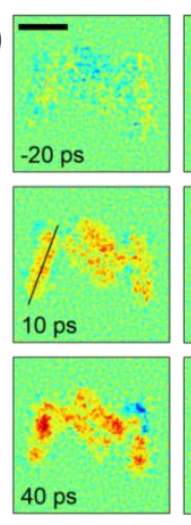

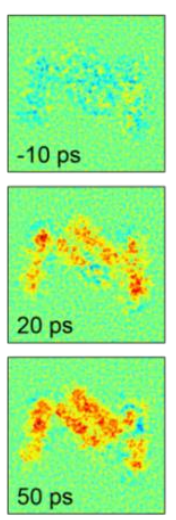
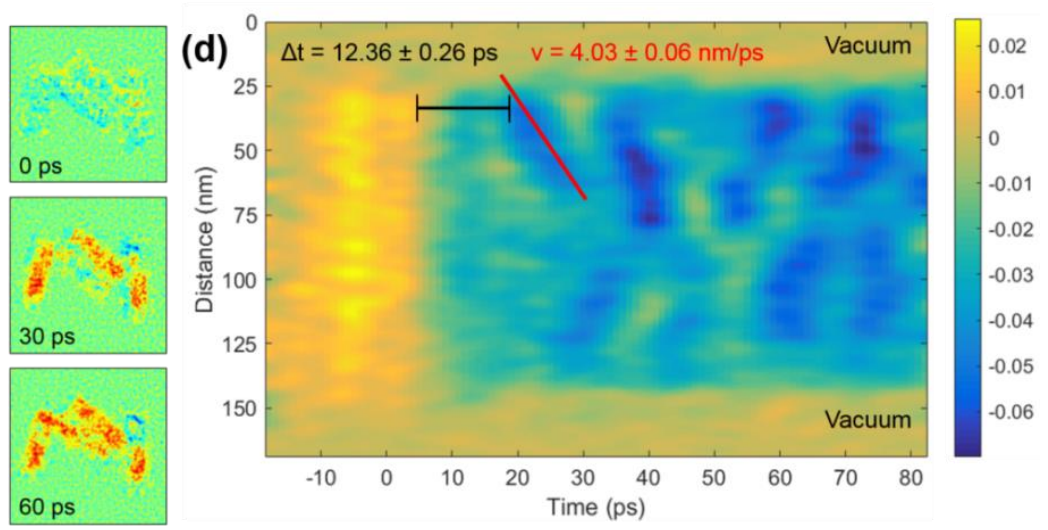

Figure 1. Incoherent and coherent structural dynamics of a cluster of plasmonic Au nanorods. (a) Color-mapped UEM bright-field image highlighting the four Au nanorods under study. The green square marks the region of the substrate used to monitor the background response in comparison to the response of each individual nanorod. Scale bar $=50 \mathrm{~nm}$. (b) Normalized counts as a function of time for each of the four nanorods shown in (a). The colors of the plots correspond to the color outlines of each particle in the UEM image. The times at which the intensity drops begin, as well as the percent intensity drop, is labeled next to each plot. The background response is shown in green for comparison. (c) Select false-colored UEM difference images of the cluster. Hotter colors indicate a decrease in image counts, while cooler colors indicate an increase. The line drawn the length of particle 1 in the 10ps frame marks the profile region analyzed to generate the space-time contour plot in (d). Scale bar = $100 \mathrm{~nm}$. (d) Space-time contour plot of particle 1 revealing the initial drop in counts followed $12 \mathrm{ps}$ later by the launch of coherent acoustic phonon modes (dark blue features). The red line marks the slope and direction of the initial acoustic phonon launched, which propagates at approximately the bulk speed of sound. Note the numbers and errors reported in (b) and (d) are from fits to the data and should not be interpreted as indicators of the level of measurement precision. 